pathogenesis of HCV-related HCC in relation to tumour progression and angiogenesis.

Method 30 patients with HCV-related cirrhosis (15 patients with histologically-proven HCC and 15 patients without HCC) and 15 healthy subjects were enrolled in the study. The severity of liver disease was assessed according to Child-Pugh classification and the Model for End Stage Liver Disease (MELD) score. The tumour stage was classified using the Cancer of the Liver Italian Program (CLIP) scoring system. Histological tumour grading was performed according to the Edmondson and Steiner's criteria and the surrounding liver tissue was examined for assessing the modified histological activity index (HAI), presence of cirrhosis and the grade of steatosis. Expressions of MIP-1a/CCL3, CD68 [a marker for tumour-associated macrophages (TAM)] and CD105 (Endoglin) [for tumour angiogenesis and determination of microvessel density (MVD)] were studied in HCC and adjacent non-neoplastic liver tissues by immunohistochemistry. Serum MIP-1a/CCL3 levels were measured using solid phase sandwich enzyme linked immunosorbant assay kit. The sensitivity and specificity of serum levels of MIP-1a/CCL3 as markers for diagnosis of HCC have been assessed by plotting a receiver-operating characteristic (ROC) curve.

Results Patients with HCV-related HCCs showed significant increases in MIP-1 $\alpha / C C L 3$ expression, $\mathrm{CD}^{+} 8^{+}$TAM count and $\mathrm{CD}_{105^{+}} \mathrm{MVD}$ in tumour tissues compared with adjacent non-neoplastic liver tissues $(p=0.0004, p<0.001$ and $p<0.001$ respectively). Serum MIP-1 $\alpha$ /CCL3 levels were significantly higher in patients with and without HCC than in healthy subjects and in HCC patients than in patients without HCC $(p<0.001)$. By plotting a ROC curve, the sensitivity and specificity of serum MIP-1 $\alpha / C C L 3$ in discriminating cirrhotic patients with and without HCC were found to be $100 \%$ and $93.3 \%$ respectively at a cut-off value of $17.5 \mathrm{pg} / \mathrm{ml}$. The MIP-1 $\alpha /$ CCL3 expression in HCC tissues showed positive correlations with serum MIP-1 $\alpha$ /CCL3 levels; tumour size, stage, histopathological grade; serum $\alpha$-fetoprotein levels and $\mathrm{CD}^{+} 8^{+}$TAM count and CD105 ${ }^{+}$MVD in HCCs $(p<0.05)$. Also, $\mathrm{CD}^{+} 8^{+} \mathrm{TAM}^{-}$count and CD105 ${ }^{+} \mathrm{MVD}$ in HCC tissues were positively correlated $(p<0.001)$. On the other hand, no correlations were found between MIP-1 $\alpha /$ CCL3 expression, $\mathrm{CD}^{+} 8^{+}$TAM count and CD105 ${ }^{+}$MVD in HCCs on one hand and serum levels of aminotransferases, Child-Pugh score, MELD score and HAI and steatosis grade in the surrounding liver tissue $(p>0.05)$.

Conclusion The CC chemokine, MIP-1 $\alpha /$ CCL3, may play an important role in the pathogenesis and progression of HCC in HCVrelated liver disease, possibly, through migration of macrophages to tumour microenvironment and enhancement of angiogenesis. MIP$1 \alpha /$ CCL3 may also serve as a potential serum biological marker and a useful therapeutic target for HCC.

\section{P53 THE COST-EFFECTIVENESS OF HCV ANTIVIRAL TREATMENT FOR INJECTING DRUG USER POPULATIONS}

\section{doi:10.1136/gutjnl-2011-300857a.53}

${ }^{1} \mathrm{~N}$ K Martin, ${ }^{1} \mathrm{P}$ Vickerman, ${ }^{2} \mathrm{G}$ R Foster, ${ }^{3} \mathrm{~A}$ Miners, ${ }^{4} \mathrm{~S} J$ Hutchinson, ${ }^{2} \mathrm{D}$ J Goldberg, ${ }^{5} \mathrm{M}$ Hickman. ${ }^{1}$ University of Bristol; ${ }^{2}$ London School of Hygiene and Tropical Medicine; ${ }^{3}$ Queen Marys University of London; ${ }^{4}$ Barts and The London School of Medicine; ${ }^{5}$ Health Protection Scotland

Introduction Injecting drug use is the main risk of HCV transmission in most developed countries. Hepatitis $C$ virus antiviral treatment (peginterferon + ribavirin) is cost-effective for patients with no reinfection risk. Concerns about reinfection and non-compliance may discourage clinicians from treating injecting drug users (IDUs), despite the potential use of treatment as prevention in this population.
Aim Using a cost-utility analysis, we examined the cost-effectiveness of providing antiviral treatment for IDUs as compared to treating ex/non-IDUs or no treatment.

Method A dynamic model of hepatitis $\mathrm{C}$ transmission and disease progression among IDUs and ex-/non-IDUs was developed, incorporating: a fixed number of antiviral treatments allocated at the mild HCV stage over 10 years, no retreatment after initial treatment failure, and potential re-infection for cured IDUs. We performed a probabilistic cost-utility analysis estimating long-term costs and outcomes (measured in OALYs) and calculating the incremental cost-effectiveness ratio (ICER) to determine the cost-effectiveness of treating IDUs as compared to treating ex/non-IDUs or no treatment for three baseline IDU HCV prevalence scenarios $(20 \%$, $40 \%$, and $60 \%$ ).

Results Antiviral treatment of IDUs is the most cost-effective option in both the $20 \%$ and $40 \%$ baseline chronic prevalence settings, with ICERs as compared to no treatment (best supportive care) of $£ 521$ and $£ 2539$ per OALY saved, respectively. Treatment of $\mathrm{ex} /$ non-IDUs is dominated in these scenarios. At $60 \%$ baseline prevalence, treatment of ex/non-IDUs or IDUs is roughly equally cost-effective; treating ex/non-IDUs is more likely to be the most cost-effective option (with an ICER as compared to no treatment of $£ 6803$ ), and treating IDUs is dominated due to the high re-infection at this prevalence. A sensitivity analysis indicates that these rankings hold even when IDU SVR rates as compared to ex/non-IDUs are halved.

Conclusion Despite the possibility of re-infection, the model projections suggest that providing antiviral treatment to IDUs is the most cost-effective policy option in chronic prevalence scenarios $<60 \%$. Further research on how HCV treatment for injectors can be scaled up, and its impact on prevalence is warranted.

\section{P54 CHASE-B (CHINESE HEPATITIS AWARENESS, SURVEILLANCE AND EDUCATION): A PILOT OF TARGETED CASE FINDING FOR HEPATITIS B VIRUS (HBV) IN THE BRITISH-CHINESE COMMUNITY}

doi:10.1136/gutjnl-2011-300857a.54

${ }^{1} \mathrm{~S}$ McPherson, ${ }^{2} \mathrm{M}$ Valappil, ${ }^{2} \mathrm{~S}$ Moses, ${ }^{2} \mathrm{G}$ Eltringham, ${ }^{1} \mathrm{C}$ Miller, ${ }^{1} \mathrm{~K}$ Baxter, ${ }^{3} \mathrm{~B}$ Brown, ${ }^{3} \mathrm{P}$ Clapper, ${ }^{4} \mathrm{~A}$ Chan, ${ }^{1} \mathrm{M}$ Hudson, ${ }^{1} \mathrm{M}$ Bassendine. ${ }^{1}$ Freeman Hospital Newcastle upon Tyne; ${ }^{2}$ HPA Newcastle upon Tyne, ${ }^{3}$ HPA Manchester; ${ }^{4}$ Chinese Healthy Living Centre Newcastle

Introduction Chronic HBV (cHBV) is a frequent cause of cirrhosis and liver cancer. Many infected individuals are unaware of their condition. Migrants from countries with high prevalence of cHBV, such as China and the Far East (seroprevalence 7-12\%), are a high risk group for cHBV. Targeted HBV screening and vaccination is recommended by the AASLD $^{1}$ and the European Liver Patients Association (ELPA $)^{2}$ in high risk groups including subjects born in endemic areas. However, there are no current UK guidelines.

Aim To apply AASLD and ELPA recommendations to BritishChinese community of North East (NE) England.

Method Members of the NE Chinese community were invited to attend screening sessions at the Newcastle Chinese Healthy Living Centre [charity registration no. 1125227]. Dry blood spots were obtained by finger prick and tested for $\mathrm{HBsAg}$ and $\mathrm{HBcAb}$ (Abbott ARCHITECT). HBsAg positive individuals were advised to undergo confirmatory testing and be referred for specialist assessment.

Results 575 subjects were screened in 10 sessions (mean age $49 \pm 17$ years, $61 \%$ female). 53 (9\%) were HBsAg positive (48\% female) indicating $\mathrm{CHBV}$. 10 of these reported being previously diagnosed with HBV, but were not under follow-up. The prevalence of $\mathrm{HBsAg}$ positivity was $7.5 \%$ when previously diagnosed individuals 